\title{
Text Interpretation and Textual Interpretive categories
}

\section{Text comprehension and genre-oriented studying of texual exactness, clarity and deepness}

\author{
Olga Valuyskaya \\ Department of English philology \\ Volgograd State University \\ Volgograd, Russia \\ olgaval@volsu.ru
}

\begin{abstract}
The article deals with the issues of text comprehension as the initial stage for further reader's interpretation. Working within the field of text interpretation the author reveals the correlation between the text comprehension, textual interpretability and the aspect of textual genres as typologies. The interplay of the aforementioned phenomena is the basis for introducing interpretive textual categories such as exactness, clarity and deepness; the latter is examined as a gradual textual category that can be embodied in the form of an operational level-organized model enabling to take into consideration genres of text.
\end{abstract}

Keywords-comprehension; interpretation; genre; interpretive categories; operational model; textual deepness; clarity; exactness

\section{INTRODUCTION}

Text comprehension issues are the ones that are closely associated with some particular humanitarian-centered theoretical problem. The concept of "comprehension" is widely discussed by educators taking into consideration cognitive factors [1]. Comprehension based on texts has certain advantages: concrete texts are finite and have precise boundaries in most cases; texts are subjected to reproduction, simulation and, thus, are available for a researcher; texts as viewed from linguistic expression plane present by themselves observation objects. The text as a coherent unit helps develop comprehension strategies [2]. The problem of text comprehension has always been interrelated with interpretation. In modern linguistics interpretation techniques are inseparably connected to text perception, understanding and comprehension. Some scholars consider the notions perception, comprehension and interpretation to be synonymous. But the differentiation between these notions is preferable; such differential approach is rational and defensible. In the most common sense, perception may be regarded as coherent reflection that appears under the direct effect of auditory and/or visual stimuli. Many scholars define text perception as a psychological process; its essence is to create a dynamic image of the text content for a recipient. It is suggested that perception should be the initial stage in the triad: perception - comprehension - interpretation; the stage that is regarded as conventional reception of a message by a device. The second stage is consequently named as insight through the analysis of the verbal form that leads to text comprehension. In this triad set, comprehension is the basic notion and it represents the ability to perceive the meaning of a text. In some research works investigators differentiate between comprehension and understanding; the reasonable differentiation is given by W. Kintch, when such elements as "perceptions, concepts, ideas, images, or emotions" enter into a process of meaningful unification of elements [3]. Text comprehension is possible without words; it is a non-verbal process, a complicated process associated with decoding abilities [4]. Interpretation presents by itself a verbalized stage, i.e. it occurs when elucidation and transition into words appear; it herewith is to be taken into consideration that first comes explanation followed by critical analysis.

Since comprehension is acknowledged as a basic notion it is noteworthy to dwell on different approaches that have been outlined in modern linguistics. In general, the following approaches to text comprehension can be outlined: logicallinguistic, systemic-semiotic, cognitive, and psycholinguistic and psycho-poetic. Logical-linguistic approach to text comprehension is a system of reasoning, its essential point is to create linguistic prescriptive systems of reasoning, the recipient aiming at comprehending of reasoning is due to know the rules of drawing conclusions and observe a principal pragmatic-semantic postulate of argumentation. Such approach is directed at scientific and scholarly texts, and therefore, it does not reveal the complexity of text comprehension and highlights one aspect of the aforementioned issue.

Systemic-semiotic approach raises the problem of developing a new conceptual scholarly apparatus which is to explain general mechanisms and abilities of cross-cultural communication in dynamics. This approach resulted in the development of the integral theory of cross-cultural communication and its focal point of a sign as a cultural phenomenon. Semiotic translation is regarded as the ground mechanism of inter-cultural communication; the conception of semiotic translation is substantiated as an infinite interpretation of signs. In terms of this approach translation is interpreted as language receding, mental interpretative activities, and inter-cultural activity. This approach takes into 
consideration intralingual translation that includes sign interpretation, and results in signification of sign actuality. The cognitive approach is fundamentally grounded on setting a mental model. Researchers in this field underline the necessity of psychic processes, without which all reasoning might stay on the level of speculative theorization.

Comprehension as text projection construction (psycholinguistic approach) is correlated with the ideas of L.S. Vygotsky [5], M.M. Bakhtin [6]. Further psycholinguistic researches in this direction led to the development of the theory that unites the process of text perception and comprehension into a coherent interaction. The basic concept of this approach is the text projection that is viewed as mental foundation, the result of the recipient's text perception which is in its turn presents the approximation of the author's text projection variant. This standpoint presupposes the system containing five constituents: an author - an author's text projection - a text body - a recipient - a recipient's text projection. A text body is the only invariable constituent of the scheme. Thus, a meta-theory of text comprehension appears within the integrative approach and the following postulates: a text body and an individual should be incorporated into a relevant culture without which the text is unlikely to exist; the leading role in text comprehension belongs to verbalized representation as the means of access to the integrated information human base; the role of a word unit is determined by its functions in an individual's lexicon; word identification takes place via its inclusion into the inner context of the prior experience (verbal and non-verbal, individual and collective), as a result there appears the united phenomenon of immediate unity of meaning and sense; text contents and its sense are as inseparable for a recipient as a word meaning and its sense; text comprehension is resulted into a cohesive emotional upheaval of a text recipient. The textual interpretation has a two-sided nature: producer-oriented and receiver-oriented (which is of a free character) [7]. Humanitarian understanding of comprehension is closely connected with interpretation as explanation which is viewed as a verbalized variant if compared to comprehension.

\section{RESEACH AND RESULTS}

Interpretation as an act and result of explanation is viewed philosophically and linguistically. The linguistic frame of interpretation points out universal and national (idioethnic) strategies. Universal interpretation strategies are founded on specific features within the theory of games that can be implemented to the description of language activity: the plane which regulates the choice from a spectrum of possible acts (alternatives are available) in a situation; the choice made is estimated from the viewpoint of its purposefulness and effectiveness; strategy outcome is not always predictable. Idioethnic strategies of interpretation being part of the national traditions are vested in: practice of perception/comprehension, interpretation as tradition of text explanation and translation and acts alike; traditions in describing native and nonnative languages; traditions of cultural and literary text reception of native and nonnative texts; grammatical and lexicographical traditions. Thus, text is one of the most complicated target matters of interpretation implementing several approaches, among them researchers choose either structural-semiotic one, or concentrate on text content-related plane. The methodological basis for the structural-semiotic interpretation presents by itself text representation as a coherent whole of interrelated elements; it means the total text with the bulk of its verbal units, structure as hierarchical relations of intratextual elements. Interpretation plurality is another aspect that is pointed out within text interpretation. Validating the potential infinity of textual interpretation in general, and fiction texts in particular, researchers associate it with the following issues: a personal aspect, i.e. coincidence or noncoincidence of some ideological, esthetic, psychological, emotional qualities of an author and a reader; impossibility of two identical interpretations, as one and the same reader can have a different interpretation after any further readings; any fiction text is infinite in its content; a literary image is multitudinous and inexhaustible in itself, and each historical epoch may be connected with a novelty in interpretation.

Thus, there arises a question whether there are as many interpretations as possible or if there may be a singular true interpretation. The latter could hardly be acknowledged as it may lead to dogmatism and it is contradictory to a research approach. A compromise lies in adopting the idea of a complex uniting adequate text interpretations. In this case the multiple character of the text is adopted, and there comes the notion of adequateness. The criterion for adequateness will be the degree of argumentativeness, proof and demonstrativeness of interpretation. The following aspects should be notable: convincing facts and reasons proving interpreter's thoughts, systematization of conclusions by means of which a new statement is derived. A text is open to multiple interpretations; a personage or an event depicted in the text may be valued or characterized by different readers and critics in diverse or even opposing ways. The matter is not within the language peculiarities of the text itself that remains stable, but within the depicted reality. Interpretation multiplicity is usually caused by extra-linguistic ambiguousness. A spectrum of interpretation typical features may be marked as a process of decoding and the result of decoding. The features of interpretation are the following: two-sidedness that presupposes the awareness and explanation for oneself and the explanation for others; multiplicity that does not mean arbitrariness; adequacy that does not equal identity; creativity that is close to co-authorship; originality which is not a goal in itself; interdisciplinary character demanding a complex approach. As a rule two types of interpretations are defined; primary or readers' interpretation and secondary or scholarly and image-making interpretations (film adaptation, theatrical adaptation, for example). Text interpretation and the papers devoted to it are linked with fiction writings mostly; and the focal point is the reader's interpretation which can be subdivided into cognitive, semantic and disobjectivating. The disobjectivating interpretation occupies the highest rank in the conditional hierarchy of interpretations and allows forming a number of mnemic patterns that can be understood as cogitative images built as a result of categorization of the idea of something or about something. The issue of text interpretation is still open in the methodology of teaching literature and teaching a second language. 
The papers that are devoted to text interpretation arise the issues of subtext, the implication (or implicit content) and the deepness of a text. The aforementioned notions are often used as synonyms and there is often no clear distinction between them. Subtext is defined as a meta-message that refers to the hidden meaning of the message [8].Subtext is often viewed as a second most significant layer of meaning and it "refers to the implied message under the text or between the lines" [9].

Differential signs of implication that distinguish it from other types of subaudition can be presented via the implementation of a logic operation. The concept of "implication" is always based on the admitted definition of a logic operation which forms a complex statement by means of a disjunction; it is revealed at the language level by the phase "if ... then" and can formally be represented as follows: $\mathrm{A} \rightarrow$ B. The assumed position is that implication is possible only in the text and can be defined as additional implied meaning derived from the ratio of appositional text units, the meaning that is not verbalized in the text body. A number of distinguishing implication features may be enumerated. Thus, one of the features is its implementation in the micro-context, the boundaries of which are determined by its referent that presents a text situation. The subtext in its turn actualizes on the macro-context level of the whole text plot, message or idea. In this case the principle of volume (immensity) and the referential correlation is distinguished. Another peculiar feature of implication is the variability of interpretation, but not a restorable unambiguity as in ellipsis, which serves as a compression of information and the elimination of its redundancy. When contrasting presupposition and text implication the latter obtains psychological predicate feature.

Proceeding from the mentioned oppositions and comparisons of different ways of subaudition one can deduce the differential features of subtext as an essential linguistic concept. The subtext peculiarities are the realization on the whole text level and the obligatory increment of the logicsubject information, which is associated with ambiguity of meaning restoration. So, subtext is a phenomenon creating additional depth of content and promoting a more complete disclosure of text messages and themes; as well as causing the emotional and evaluating reader's attitude to the narration presented in the text. Similar standpoints are found in the book by James Thomas [10]. Subtext as a meta-massage can be restored from the whole text and is inseparably connected with the context of the writing and is foreseen by the author as a method of artistic influence on readers. The hidden message of the text or the utterance may not be verbalized as it is restored from the context of fiction writing and is deliberately introduced by an author in order to achieve a literary impact on the reader; subtext as an artistic device that lies behind words gives an everlasting opportunity to enforce the expressiveness of common words, even those that might seem trivial, to show their significance, depth and strength. I assume that subtext is always associated with the author's intention and the reader's comprehension and interpretation, as well as with disclosing intended meanings. It could be the most common understanding of subtext. Subtext appears to be a property of a particular text, the result of complicated combinations of textual explicit and implicit content. After considering different features and peculiarities of subtext it can be concluded that subtext correlates with a particular text and reasonably presents a phenomenon, occurrence that might be understood via certain acts; and in this case there is the necessity to introduce the notion of "text deepness" that is associated with a interpreter's acts to explicit and reconstruct what is existing behind the text with no verbal representation in the text itself. The resultant effect of such acts represents a succession of interpretive steps, the number of which can serve as a formal factor indicating the availability of textual meaning.

The notion of "text deepness" arises from all sorts of analysis of poetical texts that are viewed as stratified systems; their interpretation brings to light all possible potential meanings organized structurally and semantically [11]. Most scholars analyzing poetic texts conclude that the deepness of poems is not transparent; and poetical texts are not always accessible to straightforward observation. Thus, there are texts with transparent deepness; they are accessible to direct observation. The conventional boundaries of text deepness are defined within textual "transparency / opacity"; intermediate phenomena are ranged between the extreme levels; intermediacy is characterized by different degree of meaning restoration, such as complete, partial and so on.

The text as a relatively independent whole exists in its form and meaning as such; the assumption that a text cannot defend itself against readers and interpreters is debatable; conventional textual self-protection is the matter of examining via some textual properties and it can be presented on a scale of conventionality of extremes: transparency and opacity. But if it is assumed that the issue of "transparency / opacity" is correlated with the problem of text defense that is aimed at making the decoding of the text unavailable for a potential eavesdropper, due to the failure to distinguish the connection between the sign used by the speaker and its referent. Proposed text defenses fall into linguistic and extra-linguistic ones; whereas linguistic text defenses include a different language or sublanguage, terminology, industry words, jargon words; extra-linguistic text defenses contain associative defense (an appeal to presupposition that is common to a speaker and addressee and unknown to an eavesdropper) and a secret code word. The situation with text defenses correlates with the issues of textual sense opacity and its decoding. In that regard a text interpretative category should be introduced, the category being contextual by its nature helps to take into consideration unambiguity / ambiguity of decoding textual sense, and to construct an operational model, i.e. a model with level-based organization allowing correlation of different texts to one or another level in accordance with its sense diversity.

The further investigation in this field is based on the classification of text categories that include the potential feature of the text to undergo an operation of interpretation; this potential feature correlates with the category of text interpretability manifesting as text accuracy, clarity, deepness, explicability / implicitness; the text interpretability is a meaningful (semantic-pragmatic) category revealing textual sense. Within the category of interpretability there are subcategories; the subcategory of text deepness is associated 
with textual sense diversity; it can also be viewed as a levelbased hierarchical organization.

The bulk of existing texts are distributed into levels in regard to the operational model of text deepness; the text distribution goes alongside with genre specificity. Therefore there is a need to address the questions of genre studies within the context, to clarify approaches to genres and to define genre as a linguistic phenomenon; and to justify genre-specific interpretability of texts. The problem of genre in modern humanitarian studies is associated with basic ideas put forward by Michail Bakhtin and he defined genres as stable and eternal types of utterances; each type of utterance or text can be characterized by certain thematic content, style (a choice of lexical, phraseological and grammatical language units), and composition structure. The diversity of genres is determined by social activities. There turns out to be the so called traditional approach to studying genres; it correlates to the theory of functional styles, which are viewed as a variation of written texts characterized by certain typical constituents; hence the choice and organization of units are in the relationship of interdependence and conditionality of the communication purpose. Besides, a functional style is regarded as a variation of standard language, the language acts in one or another socially significant sphere of human speech activities and their peculiarities are established in accordance with specific means of communication in a definite social sphere. Thus, scholarly understanding of genres emphasizes the social significance and communicative aspects.

According to the theory of functional stylistics each functional style exists in a set of ready-made models, and namely genres. The thesis assumes that organic interconnection of genre modifications; their distinct correlation to a definite style is one of the essential characteristic features of style. The issue of genre-centered approach deals with the problems of integrity of genre modifications forming one or another functional style, principles of uniting genres into groupings, the existence of genres that are of an intermediate character, the degree of genre division and demarcation, etc. Particular issues include the analysis of rigid or strict genres and their juxtaposition to loose genres within a certain functional style; analysis of oral and written genre variety [12]. The degree of scholarly development of genre and style prior investigation provides an opportunity to define the following basic ideas: a genre dominant can be found and highlighted almost in every single text; the genre dominant is expressed via a defined set of features, though genre boundaries are flexible, not stable and mutually penetrated, and genres by themselves have numerous intermediate forms. Genres are heterogeneous formations and are organized in accordance with a field-coverage principle. However, traditional stylistics admits that genre is often restricted to language units used to depict an authorial standpoint. In the course of the development of stylistics, linguists come to the conclusion that genre is a multicomponent pattern beyond the traditional understanding dealing with certain lexicon and compositional frame. Further investigations resulted in uprising of other approaches to genre description based on dynamism not statics.
The notion of genre is the focal point of stylistics, pragmatics and the theory of communicative acts. Genres are not determined merely by the formal construction of peculiar lexical, phraseological and syntactic units in regard to the typical genre composition. A text that represents a particular genre is implemented in a communicative situation, i.e. genre may be considered to be a discourse unit, but not a unit of a functional style. J. M. Swales understands genre as a communicative event with a number of communicative purposes [13]. If it is admitted that genre is a communicative event, then there should be introduced the conception of an addressee; it means that the following factors are considered: the social status of an addressee, conditions and a situation of a communicative event. The aforementioned factors taken into consideration, genre is to obtain its specificity depending on the type of the institutional communication the text refers to. A general approach to genre is correlated to the meaning of a French word, which is translated as "kind, sort, and style"; judging from this semantic meaning genre may be related to a type of text. Genre has a certain conventionalism, typical image, stereotypical modeling; these aspects allow distinguishing a pattern that is usually followed. Thus, genre is not only a pattern of text organization, but also a bulk of texts with a foreground, implemented pattern. Molding such patterns demand the investigation of a text and the culturalsocial context of textual functioning.

One of the most advantageous developments of modern anthropocentric linguistics is to study communicative genres that are socially rooted. By now philology and study of language have accumulated profound fundamental conceptions and empirical descriptions of speech genres. A speech genre is the main unit within the typology of genre studies; it is characterized as a fixed form of speech maintenance in a typical situation of human interaction. Such definition speech genre brings to light the idea of speech genre secondariness in relation to socially important situations, i.e. communicative genres that influence human behavior. A speech genre presents by itself the result of communicants' interaction; and this is as a rule a multi-act phenomenon consisting of several transactions.

The rules involved into the performance of a genre act provide a linguistic persona with freedom in a speech behavior. The freedom is primarily manifested in the choice of inner-genre strategies that depend on individual characters of linguistic personas engaged into communication. In addition, within certain genre interaction assumes the usage of speech tactics by speakers. In linguistic literature it is noted that some of the tactics within the genre speech behavior can act as independent genre units. Such mono-act units are referred to as subgenres. Several genres unite into a larger superordinate formation which is introduced as a hyper-genre. In addition to these terms, linguists introduce the concept of genre hybrid that combines the features of two genres fusing into one intermediate formation with multiple interpretations. This understanding of genre explains the communicative aspect of its essence when participants of communicative situations are characterized.

Another specific approach to genre is the issue of genre variation and distinguishing and analyzing rhetorical genres. 
Genre in rhetorical studies is understood as a stable conventional type of communicative creation, the result of speech behavior uniting in itself basic features of a rhetorical formation in their peculiar variation; the features originate on each stage of speech activity in accordance with rhetorical canonicity. Within this approach genre competence is viewed as a one of the most leading component of communicative competence; and the focal point of investigation is aimed at constructing a generalized rhetorical genre model in shape of a scheme. The foundation for such standpoint is a set of peculiar constitutive features: communicative environment, content, a certain communicative situation, members of communication, ethical form of constructing communication. In this case a number of models are possible to be established on the basis of communicative goals and described as a succession of simple sentences with the explanation of speaker's motives and mental acts defining a given type of utterances. It enables to depict communicative genres in an explication model with the priority of outlining the main communicative goal of each genre. Thus, it becomes possible to operate a rhetoric genre model interconnected with an algorithm of speaker's actions; the algorithm is acknowledged as the principal genre-forming unit. The terms "rhetorical genre" and "speech genre" are close concepts; one and the same genre can is realized as a speech genre in case of absence of specially planned, deliberate usage of speech building and introducing specified language means; in the context of conscious planning and deliberate usage of some or other means there is an evidence of a rhetoric genre. Modern rhetorical studies present a vast field of genre perspectives; it gives priorities to genre analyzing at the stage of its formation, which presupposes scrutinizing the situation that leads to genre accomplishment. The situation is a vast notion embracing a particular scenario (i.e. a place, time, and type of communication, emotional atmosphere of communicants, and their willingness to communicate, etc.); the situation takes into consideration events or motives that demand a particular speech type from a speech producer.

A genre list is of an open character; genres are invariant formations, the openness is considerably conditioned by the fact that the main term "speech genre" in genre-centered studies is of varying interpretations. The existing situation is closely associated the studying of a speech act, text type, speech strategy, speech tactics alongside with the research of speech genres as such. Defining the very essence of a speech genre as a peculiar phenomenon is impossible without comparing and correlating with adjoining phenomena and concepts. The analysis of current viewpoints in genre-oriented papers is limited to an idea that the differences in the notions of speech acts, speech acts, speech tactics, and speech strategy may be viewed from different levels of abstraction in researches.

The term of "genre" has long been used and fruitfully implemented in literary studies with many definitions. A traditional concept of genre in literary studies is a literary historically developed type of literary writing; the theoretical aspect of genre generalizes the features of a more or less broad group of writings. Further research in this question clarifies some more distinctive features of genre definition such as models of speaking and writing within the frame of cultures, textual groupings used for settling peculiar communicative tasks in peculiar communicative conditions. All points reviewed, it should be noted that genre is a real existing in a culture textual variation that is recognizable by prototypes. It is important for this research that genre as a literary code, a complex of norms and rules that informs an interpreter how to approach, examine a given text, thus enabling to comprehend and interpret it. In this respect, genre can be correlated to a certain set of conventional symbols and signal transmitting the information on textual meaningfulness. It affords grounds to examine textual genre specificity in close link with textual meaningfulness, i.e. text deepness is manifested in reference to text genre belonging.

The potential text ability to undergo interpretation is associated with the so called interpretability that is in its turn can be revealed as exactness, clarity and deepness. Interpretability is a universal textual category objectively inherent to any text; categorical constituents characterize a variety of textual object properties [14]. Text exactness is interpreted as the property and capacity to be exact. The volume of the notion "exactness" includes the following indicators: showing, depicting to the full in accordance with reality or a pattern; matching something that is predetermined and appropriate; correct in regard to a must-be pattern. Thus, the exactness is set according to the effective criteria of adequacy, truth value, verification, completeness of represented features, and compliance with a rule, pattern, and template. The exactness of a document as a text is conditioned by its communicative-pragmatic setting for the statement of the fact and regulation. The official text reflects the existing objective information on a certain sphere and directs a recipient to the required actions resulting from the knowledge of the information; the official text is clichéd and strictly structured. And not only the text structure undergoes the process of clichéing but also the specific meaningful components in their sequence. The aforementioned features form the basis for defining the exactness of the official text as the content organization in accordance of a stencil. The exactness of a scientific text is linked with the issue of choosing a term; the main demand for a scientific term is to be monosemantic. In this respect, the term could be considered to be limit of scientific exactness. The exactness of the literary text is defined by its communicative-pragmatic emphasis on the reflection of the unique way of the author's worldview in emotional-evaluative perception. This category is considered to be based on the criteria of adequateness to reality, trueness and completeness of demonstrated features. Thus, one and the same interpretative subcategory has content depending on speech genre. For example, literary speech can be viewed as the construction of the adequate image model. Exactness in the text of documents corresponds to certain formulas of presenting content, while scientific texts have the projection of exactness in the aspects of specification and development of denotative features.

Another subcategory of interpretability is defined by me as clarity; its notion contains the following characteristics: clearness, lucidity, vividness, the state of being clearly expressed, easily understood or brightly lit, transparent. Thus, 
this subcategory is defined on the criteria of brightness, visibility, certainty and distinctness. Textual clarity is an interpretative category characterizing definiteness and vividness of textual meaningfulness. Clarity is supposed to be the principal subcategory typical for scientific texts. Although the absolute index of it hardly achievable, as it is primarily associated with continuous development and deepening of scientific knowledge, its variability and incompleteness.

A special role among interpretative categories is occupied by textual deepness; it can be defined as meaningfulpragmatic correlating to unambiguity / ambiguity of restored textual sense. The semantics of lexical unit "deepness" include the following: extending far down from the top, extending far in from the outside or the front edge, i.e. there is a certain starting point; inaccessibility, invisibility and incomprehensibility are manifested with the distance from the starting point. The deepness is of gradual nature, which allows setting some level indicators of availability / unavailability on a conventional scale. In this respect I assume that each text as a meaningful unit can be leveled accordingly; textual sense is considered as available / unavailable or in other words restored / unrestored; within these limitary extremes the intermediate phenomena are placed, as there are some ambiguities in defining a certain place for them. It seems to be reasonable to consider the textual deepness correlated with the restoration of hidden sense via constructing an operational model that acquires a set of levels. It is necessary to take into account the textual feature of interpretability / noninterpretability considering the subcategory of textual deepness, which in its turn is connected with the point to what extent the restoration of the hidden sense not verbally represented in the text, is possible. As far as the understanding of the model is concerned, it is meant as a mental or sign image of any modeling object. The idea of an operational model is based on the explanation proposed by N. E. Enkvist: "A model is simplified because it aims at reproducing a selection of relevant elements of reality rather than all of the reality ... And it is operational because it should allow for the sort of manipulation that produces new data or predictions not available when the model was built" [15].

The proposed operational model has a conventional character, the boundaries of the levels are not distinctly drawn, surely there are phenomena having intermediate and transitional nature. The suggested multilevel model can be applied to the subcategory of textual deepness with the distinction of four levels, and namely: the first level containing texts with the complete restoration of hidden sense from the situation or context; the second level with hidden sense restoration that has a probabilistic character; the third level deals with the situation when the hidden textual sense is restored but it is ambiguous a priori; the forth level of the model presupposes that the hidden sense is fundamentally unrestored. Each level of textual deepness is correlated to certain types or genres of texts depending on the factor of how ambiguously or unambiguously the hidden sense can be restored. It is initially possible to start with a dichotomist division of the existing bulk of texts into interpretable and non-interpretable; the latter ones are related to the forth level, as such these texts are fundamentally non-interpretable.
Interpretable texts in their turn can obtain the category deepness of the first, second and third levels, the further triple division of interpretable texts is as follows: mono-interpretable texts are referred to the first level of textual deepness; multiinterpretable texts belong to the second and third levels of textual deepness; while interpretable "rigid" texts predominantly have the second level of textual deepness; and the third level is occupied by interpretable "flexible" texts.

The text genres help to differentiate the bulk of existing texts according to the proposed operational model and name medical prescriptions, cooking recipes, instructions and texts belonging to institutional communication (texts of juridical discourse) as mono-interpretable text with the complete textual sense restoration, such texts do not have variorum readings, and are set in accordance to a must-be pattern. In accordance with the established operational model the so called multi-interpretable texts occupy the second and third levels. Each level is characterized by a different number of interpretive steps to restore textual sense. Texts of newspapers, magazines and scientific publications are multiinterpretable and rigid; and they can be placed to the second level. The ambiguity in textual sense restoration of such texts is associated with their genre peculiarities typical for media and science discourses. Demands for such text genres are as follows: officialism, objectiveness, impartiality. All genre multiplicity of media discourse is traditionally divided into informational, analytical and publicistic. The number of interpretive steps that is necessary for the interpreter to restore the textual hidden sense are fewer for informative genres of texts, whereas the number of interpretive steps increases to restore the hidden sense of publicistic genres, since informative genres are mostly factual by nature, but publicistic genres (pamphlets, essays, etc.) can be metaphorical and conceptually significant. A relative transparency of textual sense is preserved in either of genres. As far as scientific texts are concerned, the principal demand for the texts is unambiguity which assigns relative rigidness while interpreting; science presents a sphere for the most transparent and monosemantic language usage, but the potential field for ambiguity and ambivalence in scientific text interpretation is constantly being produced due to further development of scientific knowledge.

The third level of the operational model of textual deepness deals with flexible interpretable texts; it embraces fiction texts (prose and poetry), with the exceptions for modernist and postmodernists writings. This is the most numerous and complicated genre level of textual deepness as far as genre variation is concerned. The bulk of texts united here have some common peculiar features that correspond to a larger number of interpretive steps if compared with the previous levels; besides these texts display a relative interpretative flexibility and give freedom and the possibility of self-expression to the interpreter; a fiction text viewed as a specific producer of hidden senses gives an opportunity to the interpreter to restore textual deepness beyond verbalized information; each new reading of the same fiction text adds new understanding and further evaluation. I think that the interpretation of a fiction text must have a nucleus that determines the conceptual textual dominant from which the 
hidden text is restored. It can be a symbol, an extended metaphor, allusion, or a precedent text and a conceptualized notion. The textual deepness of fiction texts depends on such features as interpretive flexibility, multiplicity of textual imagery.

On the assumption of the operational model of textual deepness the final forth level is considered to be the one, which contains non-interpretable texts of modernist and postmodernist poetry; the textual sense of these text is fundamentally unrestored, as these are texts-puzzles, textsriddles or even cryptograms with opaque textual sense due to unlimited experimenting and innovating with genre, form and content. The interpreter has to seek for interpretive steps that help restore traditional text standard and then to decode opaque sense.

\section{CONCLUSIONS}

The basis ground for text interpretation consists in textual availability to mass readers, possibility of expressing criticism to text content, verbalization of text comprehension.

Text comprehension presents by itself the integrity of interpreter's conceptualization of his/her mental actions; the initial stage of comprehension is textual comprehensibility.

Interpretation is viewed as the process and the result, and possesses a complex of features: two-sidedness, i.e. there is an explanation and/or substantiation for self-awareness and explanation and/or substantiation for others; multiplicity, adequacy, creativity, originality, interdisciplinarity (text linguistics, genre studies, hermeneutics), stepping character which is represented by means of a succession of interpretive steps. Interpretation multiplicity is one of the main features; multiplicity is supported by: a personal aspect and possible incongruity of ideological-aesthetical, psychological and emotional traits of different interpreters; potential textual sense endlessness, in a fiction text in particular; multidimensionality of a word-picture; diachronic mobility of elucidation of the same text.

Text interpretability is an inseparable textual feature and can be manifested as exactness, clarity and deepness. Textual deepness largely depends on genre, which in its turn is an indicator that defines interpreter's strategy and provides the restoration of a hidden sense. Textual deepness is of gradual character and can be represented as a multi-level operational model that appears to be associated with such textual aspects as genre and type.

\section{References}

[1] J. Oakhill, K. Cain, C. Elbro, Understanding and teaching reading comprehension: a handbook, London and New York: Routledge, Taylor \& Francis Group, 2014, pp. 22-54.

[2] J. Moreillon, Collaborative strategies of teaching reading comprehension: maximizing your impact, Chicago: American Library Association, , 2007, pp.11-14.

[3] W. Kintch, Comprehension: a paradigm for cognition, Cambridge: Cambridge University Press, 1998.

[4] J. V. Hoffman, Comprehension is not simple: considering the persisting dangers in the simple view of reading comprehension," in "Handbook of research on reading comprehension, 2nd ed., New York and London: Guilford Press, 2016, pp. 57-69.

[5] L. S. Vygotsky, Thought and language. Trans. Cambridge, Mass., and New York : MIT Press and John Wiley \& Sons, 1964.

[6] M. M. Bakhtin, Speech genres and other late essays, Trans., Austen: University of Texas Press, 2010.

[7] R. G. van de Velde, Text and thinking: on some roles of thinking in text interpretation, Berlin and New York: Walter de Gruyter, 1992.

[8] R. Ballon, Breathing life into your characters: how to give your characters emotional and psycological depth, Cincinnati: Writer's Diagest Books, 2009.

[9] L. E. Catron, S. Shattock, The directors vision: play direction from analysis to production, Long Grove: Waveland Press, 2015.

[10] J. Thomas, Script analysis for actors, directors and designers, New York and London: Focal Press, 2013.

[11] T. Eagleton, Literary theory: an introduction, Oxford: Blackwell Publishers, 2011.

[12] K. Wales, A dictionary of stylistics, 3rd ed., New York: Routledge, 2014.

[13] J. M. Swales, Genre analysis: English in academic and research settings, Cambridge: Cambridge University Press, 1991.

[14] O. R. Valuyskaya, "Interpretativnost kak potentsialnoe svoystvo teksta" [Text interpretativity as a potential feature], Science Journal of Volgograd State University Linguistics, vol. 11, No 2, pp. 210-215, Volgograd: VolSU Pub., 2012

[15] N. E. Enkvist, "Text linguistics for an applier: an orientation," "Writing across languages: analysis of $\mathrm{L}_{2}$ Text," Massachusets-San Juan: Addison-Wensley, 1993, pp. 23-43. 